\title{
HIGHLIGHTS
}

\section{Conserved assembly of polarity complexes}

DIX protein
domains...
have a
conserved
role... in
enabling
concentration-
dependent
protein
polymerization
and the
assembly of
cell polarity
complexes

Cell polarity is essential for the development of multicellular organisms, and its establishment depends on the coordination of local and tissue-level or organismal-level cues. These directional cues are usually translated into subcellular responses, for example the local formation of protein complexes. Given the essential role of polaritydetermining systems, Weijers, Bienz and colleagues investigated whether polarity mechanisms may be shared between plants and animals. In Cell, they now report that DIX protein domains - present in the plant SOSEKI proteins that the authors analyse in this study - have a conserved role, in animals and plants, in enabling concentration-dependent protein polymerization and the assembly of cell polarity complexes.

Multicellularity has evolved independently in plants and animals, and several plant-specific proteins display polar localization and have been linked to polarity. Many of these, however, function in a cell-dependent or tissue-dependent manner and are probably readouts or effectors of polarity systems, rather than components of 'universal' polarity-generating pathways, which remain elusive in plants. The authors

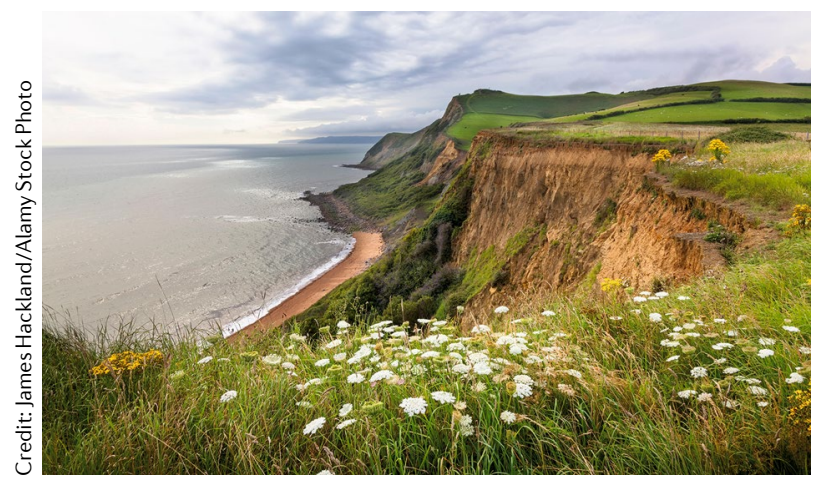

recently identified the SOSEKI proteins - in Arabidopsis thaliana - as a family of five paralogues (SOK1-SOK5) that are polarly localized in multiple cell types throughout development. Their polar localization was reported to depend on two conserved domains: a central domain required for membrane localization at polar edges and an $\mathrm{N}$-terminal domain that is required for more focused localization at these edges. The latter was proposed to contain a DIX domain-like fold, which is found in animals in the Dishevelled (DVL) protein. DVL has a key role in planar cell polarity as it transduces WNT signals in both canonical and non-canonical WNT signalling pathways; polymerization of its DIX domain mediates the formation of WNT signalosomes.

First, by analysing an RNA-seq dataset (OneKP) that encompasses transcriptomes from thousands of plant species, as well as $>100$ curated genome sequences, the authors found that SOSEKI-related sequences are widespread throughout land plants. Moreover, SOSEKI proteins were polarly localized in bryophyte species (the moss Physcomitrella patens and liverwort Marchantia polymorpha), together suggesting that SOSEKI genes have an important role in land plants, and that the polar localization of the proteins is an ancestral property.

Purified DIX domains from the SOSEKI proteins formed oligomers, via head-to-tail interactions, similarly to their animal counterparts, and higher concentrations led to larger oligomers. Thus, in addition to displaying structural similarities, the plant and animal DIX domains seem to be biochemically equivalent.
Mutagenesis of the DIX domain in the AtSOK1 protein reduced its polarization, and this resulted in alterations in the cell division planes in developing roots. Importantly, substitution of the DIX domain in DVL2 with the DIX domain from AtSOK1 led to a protein that was capable of mediating the formation of WNT signalosomes in cultured human cells; and AtSOK1 in which the DIX domain was substituted with that of DVL2 was capable of forming polymers with polar localization and had the same effect on orienting cell division planes in Arabidopsis roots as did the endogenous protein. Thus, SOSEKI and DVL2 DIX domains are functionally interchangeable and important for polarization in animals and plants.

Lastly, it is known that the increased local concentration of DVL is necessary for the recruitment of its low-affinity signalling effector Axin, but the effectors of SOSEKI proteins are unknown. Pull-down assays identified ANGUSTIFOLIA (AN) - the function of which is poorly defined but associated with cell shape, cell division orientation and organ shape - as a possible effector. AN localized at cell edges and was shown to require the DIX-dependent AtSOK1 polymerization for its recruitment to membranes and polar localization.

Thus, although specific polarity proteins have evolved independently in plant and animals, the assembly of polarity complexes follows similar principles that depend on the polymerization of conserved DIX domains, which phylogenetic analyses traced back to basal eukaryotes.

Kim Baumann

ORIGINAL ARTICLE van Dop, M. et al. DIX domain polymerization drives assembly of plant cell polarity complexes. Cell 180, 427-439 (2020) RELATED ARTICLES Yoshida, S. et al. A SOSEKIbased coordinate system interprets global polarity cues in Arabidopsis. Nat. Plants 5, 160-166 (2019) Butler, M. T. \& Wallingford, J. B. Planar cell polarity in development and disease. Nat. Rev. Mol. Cell Biol. 18, 375-388 (2017) 